\title{
Nanoplasmonic Filters for Hollow Core Photonic Crystal Fibres
}

\author{
Peter Reader-Harris* and Andrea Di Falco* \\ SUPA, School of Physics and Astronomy, University of St Andrews, North Haugh, St \\ Andrews, KY16 9SS, United Kingdom \\ E-mail: pjrh@st-andrews.ac.uk; adf10@st-andrews.ac.uk \\ Phone: +44 (0)1334 463165. Fax: +44 (0)1334 463104
}

\begin{abstract}
We demonstrate a method to add photonic functionalities to the tip of hollow photonic crystal fibres, for ultra-compact and integrated lab-on-fibre applications. Specifically we present an angularly robust passband filter that is directly applied to the facet of a fibre, without adhesive or etching. The filter exploits the engineered plasmonic response of metallic features realised on a polymeric membrane and works over a range of 10 degrees, with a passband of $50 \mathrm{~nm}$, centred at a wavelength of $800 \mathrm{~nm}$. This result extends the available functionalities offered by the lab-on-fibre methodology to photonic crystal fibres, as well as providing a reversible method of functionalizing any type of fibre.
\end{abstract}

Keywords: Lab-on-fibre, photonic crystal fibres, flexible photonics, surface plasmon resonances

\footnotetext{
${ }^{*}$ To whom correspondence should be addressed
} 


\section{Keywords}

Lab-on-fibre, photonic crystal fibres, flexible photonics, surface plasmon resonances

Optical fibres are a long established technology for light transport, but are increasingly being used for more exotic purposes, to take fibres beyond point to point light delivery. Various ways to introduce additional functionality to fibres exist, including the well-established techniques of fibre Bragg gratings and photonic crystal fibres (PhCF). The latter, in particular, have become extremely useful photonic elements, as they combine accurate light dispersion management with high field localisation. PhCF have been used for very high numerical aperture devices, ${ }^{1}$ single mode operation at all wavelengths ${ }^{2}$ and polarisation control. ${ }^{3,4}$ In addition, $\mathrm{PhCF}$ are ideal candidates for strong non-linear processes like the generation of supercontinuum light, ${ }^{5,6}$ as well as the propagation of solitons. ${ }^{7}$ A peculiar type of fibre is the hollow core photonic crystal fibre $(\mathrm{HCPhCF})$. In this case, light is confined in air by reflection from the band gap of an array of holes surrounding the hollow light-guiding region. ${ }^{8}$ This type of fibre is particularly useful in sensing applications, ${ }^{9}$ with either gases or liquids flowing through the hollow sections. Sensing can be performed by a number of different methodologies, including near infrared (NIR) absorption spectroscopy ${ }^{10}$ and fluo-

rescence spectroscopy, ${ }^{11}$ where the light signal is coupled from the fibre into a number of external components which process the signal. Integrating these often bulky components directly on the fibres could increase the portability and practical impact of PhCF sensors at reduced costs.

An elegant solution to this requirement is offered by the Lab-on-Fibre (LoF) approach. This emerging technology is a complementary method for the control of light behaviour in fibres, adding functionality to their surface, usually the facet. ${ }^{12,13}$

LoF fabrication techniques can be classified into the transfer method ${ }^{14}$ and the direct writing method. ${ }^{15}$ The latter uses the same lithographic processes to define nano-features on planar wafer substrates, adapted to more challenging topology of the fibre tip. The transfer method decouples the choice of fibre and the method of fabrication, by realising 
the functional element separately from the fibre and then combining them later. Flexible photonic membranes ${ }^{16-19}$ are exceptionally well suited for this task, as they are compliant to the fibre facet, free standing, they can be easily replaced, and can be made cheaply. Examples of the opportunities arising from these unique properties include rolled up multilayers, ${ }^{20}$ the fabrication of nano-apertures in a flexible membrane, ${ }^{21}$ as well as stretch tuning ${ }^{22}$ and flexible negative refractive index materials. ${ }^{23}$

In this paper we present the use of flexible photonic membranes as a general method to reversibly functionalize the facet of any type of fibre, including where the facet is "just air", as in the case of $\mathrm{HCPhCF}$.

Specifically, we focus on a design inspired by the guided mode resonance (GMR) filter, based on the interaction of light confined in a waveguide with an adjacent grating. In our case the membrane itself acts as a waveguide, whereas the grating is realized by patterning an overlaying gold layer, supporting surface plasmon polaritons (SPPs). This coupling can create narrow linewidth filters, ${ }^{24}$ typically at the cost of a strong angular dependence. This issue can be solved by mounting the filter on top of a fibre collimator, ${ }^{19}$ although this implementation reduces the compactness of the filter. Alternative published methods for creating angularly robust GMR-type filters at telecom wavelengths use doubly periodic gratings systems on a multilayer. ${ }^{25} \mathrm{~A}$ theoretical investigation with a doubly periodic structure incorporating a grating on both sides of the membrane has shown similar results in the mid-IR. ${ }^{26}$

Here we present a solution based on a single period grating, which is angularly robust enough to filter efficiently a fibre with a numerical aperture (NA) of 0.17 (10 degrees).

Remarkably, our filter is the simplest amongst the whole family of metasurfaces, ${ }^{27}$ but demonstrates that the platform is a robust way to create an arbitrary photonic response on the tips of Photonic Crystal fibres, thus bringing a new design paradigm to the field. 


\section{Results}
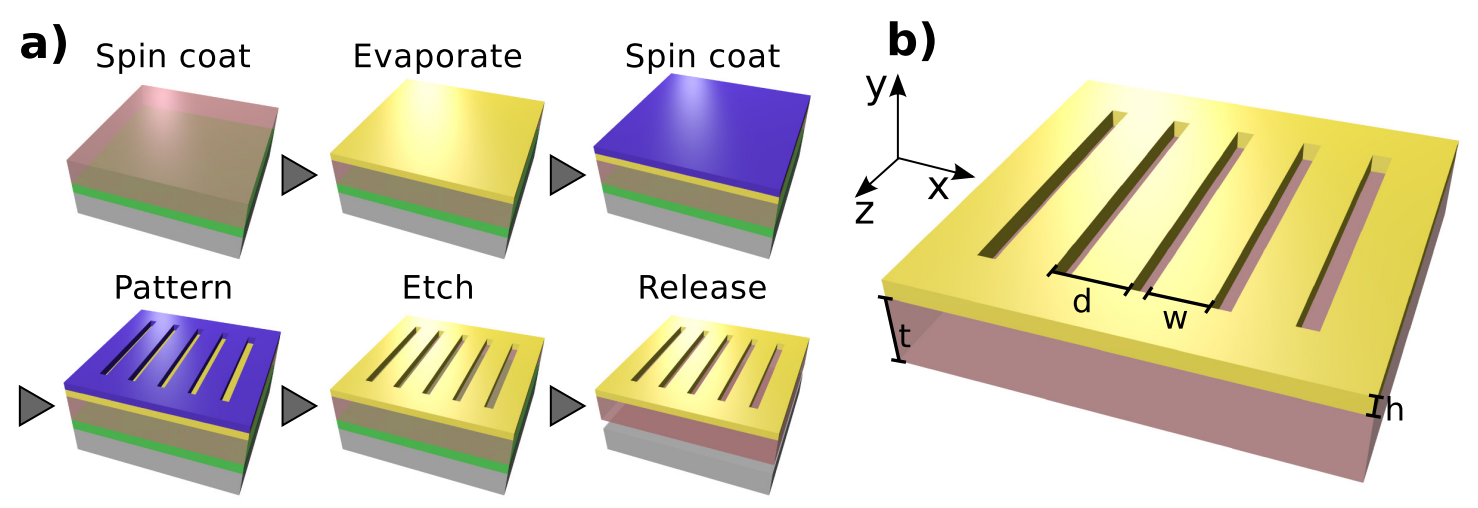

Figure 1: a) Sketch of the fabrication protocol. b) Sketch of the final membrane with relevant geometrical parameters.

The membrane was fabricated with the process illustrated in Figure 1a). A sacrificial layer and a polymer layer are deposited via spin coating on a rigid substrate, followed by the evaporation of a layer of metal. A positive tone photoresist is patterned via electron beam lithography and this pattern is transferred into the gold via reactive ion etching. Finally, the sacrificial layer between the substrate and the membrane is dissolved and the membrane in released. If necessary, the membrane can be mounted onto another object, or a frame. The fabrication details can be found in the methods section. Figure 1b) shows the relevant geometrical parameters, with typical membrane thickness of $260 \mathrm{~nm}$ and gold thickness of $40 \mathrm{~nm}$. On the metal we engraved an array of slits of $150 \mathrm{~nm}$ width and periodicity of 600 nm. However, the fabrication and manipulation protocol used here is not specific to this pattern. We have fabricated membranes in this fashion with different patterns, as well as with continuous gold, and without any gold.

The band structure relative to our geometry is shown in Figure 2 b), as calculated by a finite element analysis package, for both TE (electric field parallel to the $\mathrm{z}$ axis, red open circles) and TM polarizations (magnetic field parallel to the $\mathrm{z}$ axis, black full circles). The TE band structure does not support surface plasmons and was reported for completeness. Additionally, the TE modes of the waveguides can be tuned fairly independently of the TM 

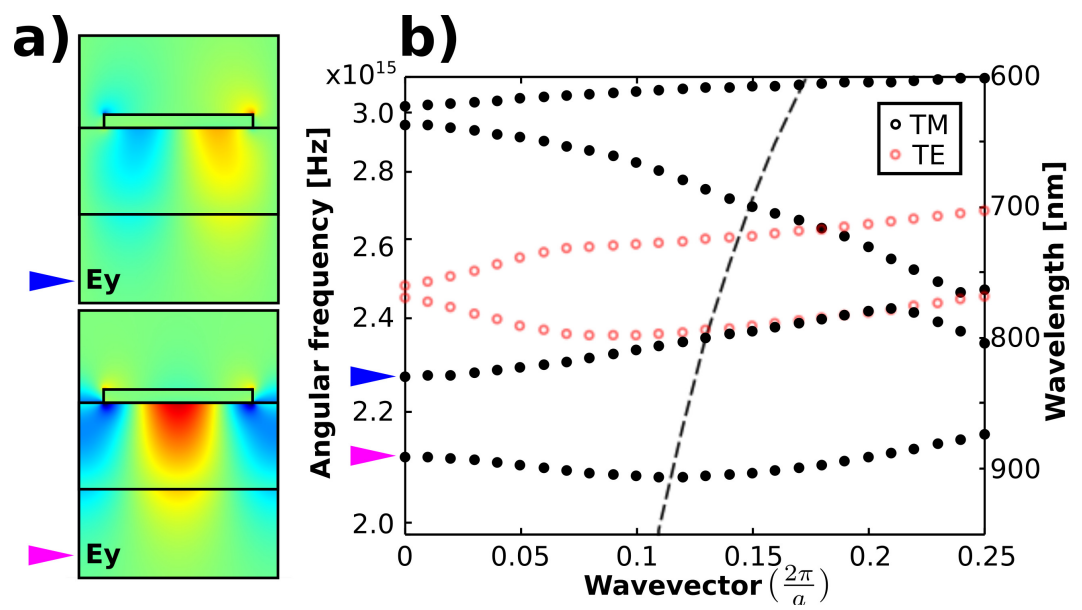

Figure 2: a) The electric field profile (Ey) for the anti-symmetric (top, blue arrow) and symmetric (bottom, pink arrow) bands at $k=0 \mathrm{~b}$ ) Band structure for the fabricated sample for TE (red open circles) and TM (black full circles) polarizations. The dotted line shows the wavevector for each wavelength corresponding to $10^{\circ}$ incidence from normal.

resonances by changing the thickness of the membrane alone. To implement an angularly robust filter we require a flat band structure for a range of wavevectors around the normal incidence value $(k=0)$, at the operation wavelength. This can be obtained by engineering the anti-crossing of the the bands of the supported states. ${ }^{28}$ The symmetry of the bands is crucial to determine the coupling efficiency of light incident on the filter. ${ }^{29}$ Figure 2a) shows the $y$-component of the electric field in the xy plane for two contiguous bands in the working wavelength region. Light incident on the sample couples efficiently only to the antisymmetric band centred at $800 \mathrm{~nm}$, whereas the coupling to symmetric bands (higher and lower frequencies) is not as efficient.

This is also confirmed by the angularly resolved transmission spectra for the TM polarization shown in Figure 3. The simulated transmission map (panel a)), obtained with rigorous coupled wave analysis (RCWA), coincides with the experimental data (panel b)), obtained on the free standing membrane. The transmission map is in perfect agreement with the band structure of Figure 2 and shows excellent angular robustness at $800 \mathrm{~nm}$, which is sufficient to accommodate the NA of the chosen HCPhC fibre. In Figure 2 we marked the incident angle of $10^{\circ}$ with a dashed line, as a guide for the eye. 

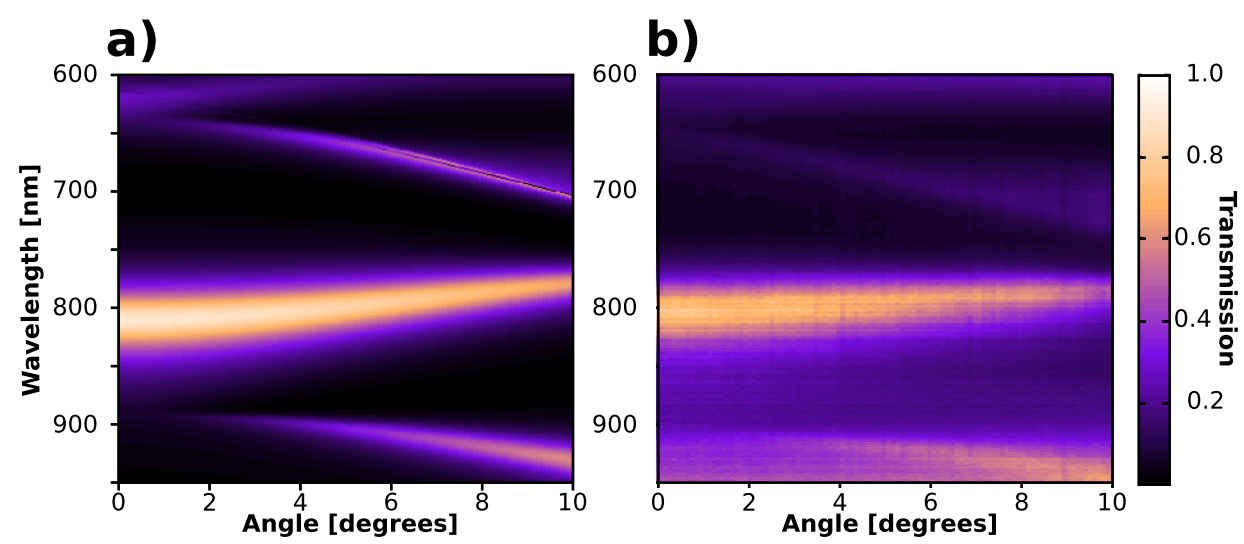

Figure 3: a) RCWA simulation of the angularly resolved transmission spectrum of the filter. b) The experimentally measured angularly resolved transmission spectrum.

The filter membrane was then mounted on the tip of the fibre as sketched in Figure 4a). A small PDMS collar was fabricated using an empty fibre ferrule. This assembly allowed the sample to be placed in direct contact with the fibre facet reversibly, so that the same membrane could be used on different fibres. The patterned area of the photonic membrane was centred on the collar with a micropositioner. The membrane was in contact only with the collar and fibre during the measurements, as in the figure. If required, the membrane could be glued permanently in place. Figure $4 \mathrm{~b}$ ) shows an optical micrograph of a typical membrane being applied to a photonic crystal fibre, the facet of which is shown in the Scanning Electron Microscope (SEM) image shown in panel c). Panel d) shows a SEM image of the nano-slit grating. The interference fringes on top right of panel b) are caused by a fibre facet which is not completely planar, possibly due to imperfect cleaving, which prevents perfect contact with the membrane on the corner. Remarkably, the flexible nature of the membrane permits anyway a good contact on the core area of the fibre, despite the non-planar facet.

The pass band of the filter was centred at $800 \mathrm{~nm}$, to overlap with the transmission band of the used $\mathrm{HCPhCF}$. The resulting transmission spectrum, normalised to the spectrum from the unfiltered fibres, is shown in Figure 5, along with RCWA simulations for the same filter geometry, taking into account the range of input angles. Using the angular spread as a fitting 

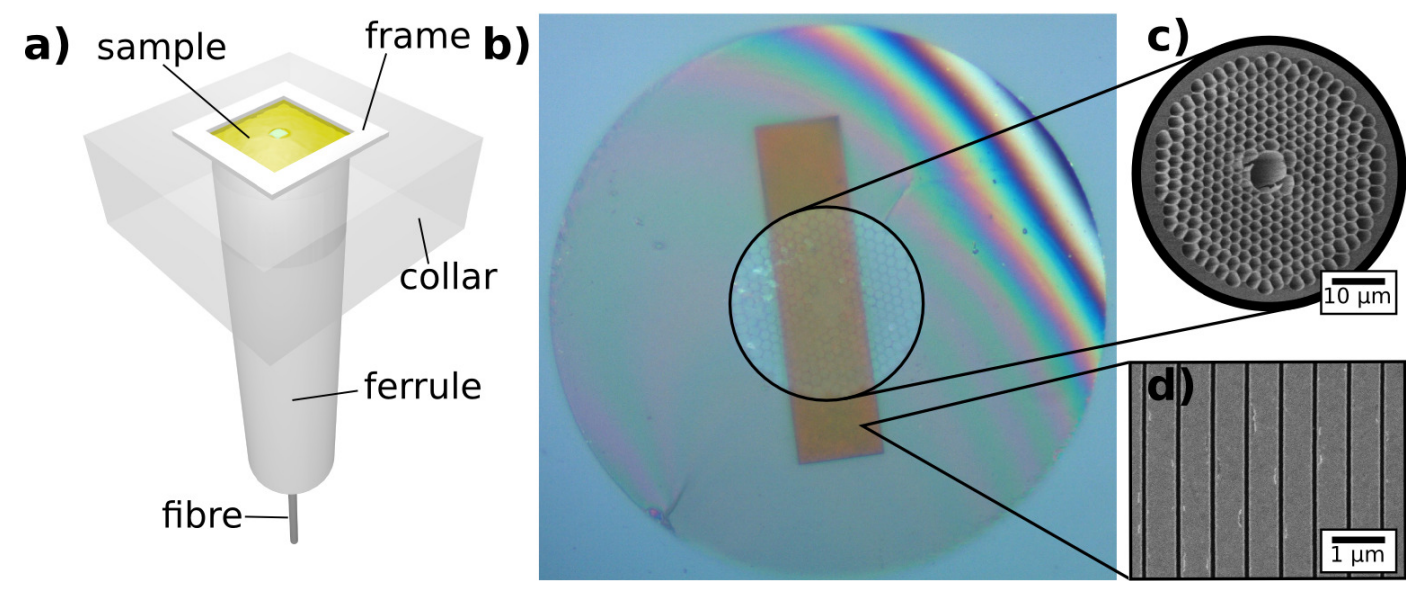

Figure 4: a) The assembly of the complete lab-on-fibre structure. b) Optical micrograph of the $\mathrm{HCPhCF}$ with a gold nano-structured membrane applied. c) SEM picture of the $\mathrm{HCPhCF}$ facet used. d) SEM picture of the gold nano-slit grating.

parameter we found that the RCWA simulations fitted best for a spread of 10 degrees, which corresponds to an NA of the fibre of 0.17. The resonance has a Full-Width Half-Maximum (FWHM) of $51 \mathrm{~nm}$. This design also permits polarization control, with the SPPs coupling with TM light. The small dip in the TE transmission spectrum, at $760 \mathrm{~nm}$, is caused by the small band gap shown in the TE band structure (red) of Figure 2. Using a thicker gold layer increases the polarization contrast, although at the possible expense of increased losses.

From a practical point of view, we note that despite the complete filter being less than $300 \mathrm{~nm}$ thick, the membrane does not require any special handling procedures beyond that of standard optical components, once mounted into the frame. Once framed, we used a micropositioner to align the patterned area of the membrane with the core of the fibre. Patterning a larger area of the membrane could trivially lift this requirement. The fabrication yield of the flexible metasurface is practically $100 \%$, and mounting the fibre into a ferrule prevents the membrane from being pierced during assembly. The filter is reusable, and can be transferred from fibre to fibre, without observable loss of performance. 


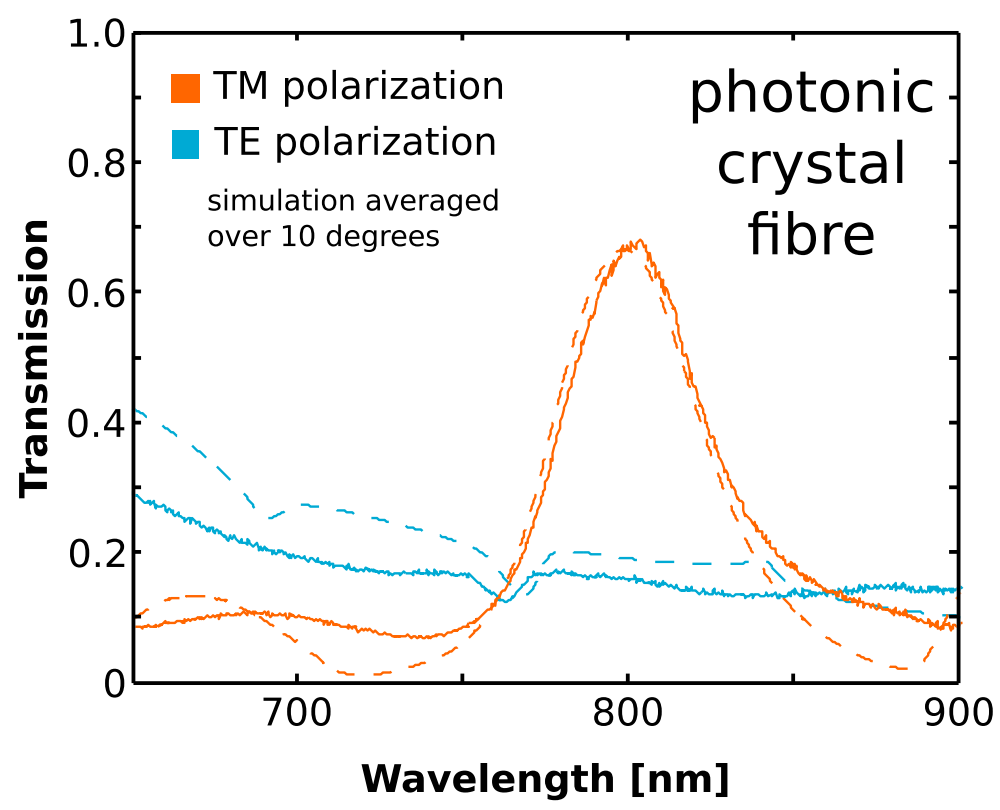

Figure 5: The measured transmission spectrum for both TE and TM polarization as mounted on two types of optical fibre (solid), overlayed with RCWA simulation, taking into account the spread of angles from the fibre (dashed).

\section{Discussion}

The use of flexible photonic membranes for lab-on-fibre applications has a number of desirable properties. The transfer of a flexible membrane enables a good contact onto the fibre facet, without reliance on adhesive or Van der Waals' forces. Rigid photonic crystals have been transferred onto a fibre facet, using epoxy as a glue, ${ }^{30}$ but this is essentially irreversible. Our approach grants the reusability of the membrane and of the fibre, which is not permanently modified by the application of the filter. However it is possible to wrap permanently the membrane at the end of the fibre, thus without using the frame, ferule and PDMS collar.

In order to create angular robustness in the GMR-type filter we used metal, thus introducing losses that are detrimental from a bandwidth point of view. A possible solution is to replace the metal with a high index dielectric, while retaining the flexible nature of the substrate, possibly using a photonic crystal design. However, it is typically easier to get the large anti-crossing required to generate angular robustness with SPP-type propagation than with all-dielectric waveguide systems. Additionally it is easier to conceive scaling up the 
fabrication of metal-based flexible filters than their all-dielectric counterparts.

$\mathrm{HCPhCF}$ have the unique ability to pass fluids through the hole which runs their length, enabling a new pathway for LoF integration into sensing experiments. An example of this is surface enhanced Raman spectroscopy (SERS) which has been demonstrated with a hollow core photonic crystal fibre as the substrate. ${ }^{31,32}$ A filter could be usefully employed to eliminate the pump light from the transmitted beam in such a SERS experiment, which would be a step forward to a compact, integrated system for SERS, rather than the bulky apparatus used today. Additionally, in HCPhCF the shape of the flexible fibre mounted device could be tailored by varying the applied pressure of fluid in the fibre, thus tuning the LoF properties.

The filter presented here is just one example of the flexible photonic membrane platform. Other applications could include fibre mounted optomechanics, or beamshaping. In the case of optomechanical systems, light from the fibre can create vibrational modes in the ultrathin membrane by radiation pressure. Direct beamshaping of a fibre's output is a possibility with patterned surfaces, as has already been demonstrated in free space at NIR frequencies. ${ }^{33,34}$ Generating the desired eigenmode straight from the fibre, including Bessel beams or orbital angular momentum modes, could create new applications for optical trapping or coupling to external devices. Structured fibre facets have already shown their use in endoscopy, ${ }^{35}$ and we see the ability to reversibly add sensing, and other, elements to endoscopes as a key area for lab-on-fibre to impact.

\section{Conclusion}

We have demonstrated a fabrication protocol to functionalize the facet of any fibre. This permits to merge the promising potential of the lab-on-fibre protocol to the already rich physics of hollow photonic crystal fibres. We have shown the versatility of this technique by creating a reusable angularly robust spectral filter. This result could lead to the demonstration of a new class of compact, integrated fibre based devices. 


\section{Methods}

Bandstructure Simulation. The simulation of the band structure of periodic metallic nano-structures is typically complicated in the IR/visible regime by the highly dispersive metal response. To compute the band structure we used a wave vector marching technique on COMSOL Multiphysics. We started by calculating the eigenfrequencies at $k=0$, and used them as the initial inputs to calculated the eigenmodes for small increments of $k$. As long as the steps in $k$ are small enough, the dispersion in the optical constants can be assumed to be small.

Device Fabrication. The flexible photonic membrane was fabricated according to a method adapted from ref. 16. A silicon wafer was used to provide the rigid, planar support for the fabrication steps. It was spin coated with Omnicoat, a release layer from Microchem Corp. at $1000 \mathrm{rpm}$ and baked at $230^{\circ} \mathrm{C}$ for $1 \mathrm{~min}$. Then a diluted epoxy-based polymer (SU8 2000.5, from the same company, 1:2 in cyclopentanone) is spun to a thickness of about $260 \mathrm{~nm}$ at a speed of $1000 \mathrm{rpm}$, before baking at $100^{\circ} \mathrm{C}$ for 5 mins. The SU8 is then cured for 2 mins with ultraviolet light, and baked at $100^{\circ} \mathrm{C}$ for a further 2 mins. A $40 \mathrm{~nm}$ thick gold layer was then evaporated with an electron beam evaporator, and patterned via electron beam lithography (30 kV with a dose of $800 \mathrm{pAs} / \mathrm{cm}$ ) using ZEP520A, from Zeon Corp. as photoresist, spun at $5000 \mathrm{rpm}$, and baked for 20 mins at $140^{\circ} \mathrm{C}$. Xylene at $23^{\circ} \mathrm{C}$ was used to develop the ZEP520A for 45 seconds. Etch back was then achieved with an Argon plasma in a reactive ion etcher, for 9 mins at a DC bias of $-333 \mathrm{~V}$. Any left over ZEP was cleaned by trichloroethylene in an ultrasonic bath. The initial Omnicoat layer was then released using $N$-methyl-2-pyrrolidone at $60^{\circ} \mathrm{C}$. The membrane was then transferred to a water bath where it could be hydrophobically suspended on the water's surface, and subsequently manipulated or mounted into a frame or onto another object. 
Sample Measurement. The samples were measured first in a free standing configuration in order to get the angularly resolved spectrum of Figure $2 \mathrm{~b}$ ). The sample was directly illuminated by a polarised Koheras SuperK Compact supercontinuum laser. An objective was used to imaged the sample in transmission onto a punctured screen, such that the light illuminating the patterned area of the sample went through to an Ocean Optics 2000+ USB spectrometer. For the spectra in Figure 5, the sample was then mounted onto the fibre and collar by a micrometer drive, and the fibre was illuminated at one end by an unpolarised halogen lamp source. The sample coated end was imaged with a $20 \times$ objective into an Ocean Optics 2000+ USB spectrometer. A polariser was place between the objective and the spectrometer, so as to observe the polarisation dependence of the filter. In both cases (free space and fibre measurements) the normalisation spectra were taken for both polarisations with the same setup without the sample present. The fibre used was a hollow core photonic crystal fibre from NKT Photonics: HC-800-02.

\section{Acknowledgement}

The authors acknowledge support from EPSRC (EP/I004602/1).

\section{Supporting Information Available}

This material is available free of charge via the Internet at http://pubs.acs.org/.

\section{References}

(1) Wadsworth, W.; Percival, R.; Bouwmans, G.; Knight, J.; Birks, T.; Hedley, T.; Russell, P. St. J. Very High Numerical Aperture Fibers. IEEE Photonics Technol. Lett. 2004, 16, 843-845.

(2) Birks, T. A.; Knight, J. C.; Russell, P. St. J. Endlessly single-mode photonic crystal fiber. Opt. Lett. 1997, 22, 961-963. 
(3) Suzuki, K.; Kubota, H.; Kawanishi, S.; Tanaka, M.; Fujita, M. Optical properties of a low-loss polarization-maintaining photonic crystal fiber. Opt. Express 2001, 9, 676-680.

(4) Kubota, H.; Kawanishi, S.; Koyanagi, S.; Tanaka, M.; Yamaguchi, S. Absolutely Single Polarization Photonic Crystal Fiber. IEEE Photonics Technol. Lett. 2004, 16, 182-184.

(5) Dudley, J. M.; Genty, G.; Coen, S. Supercontinuum generation in photonic crystal fiber. Rev. Mod. Phys. 2006, 78, 1135-1184.

(6) Travers, J. C.; Rulkov, A. B.; Cumberland, B. A.; Popov, S. V.; Taylor, J. R. Visible supercontinuum generation in photonic crystal fibers with a $400 \mathrm{~W}$ continuous wave fiber laser. Opt. Express 2008, 16, 14435-14447.

(7) Travers, J. C. Blue solitary waves from infrared continuous wave pumping of optical fibers. Opt. Express 2009, 17, 1502-1507.

(8) Cregan, R. F.; Mangan, J. C.; Knight, J.; Birks, T.; Russell, P. St. J.; Roberts, P. J.; Allan, D. C. Single-Mode Photonic Band Gap Guidance of Light in Air. Science 1999, 285, 1537-1539.

(9) Cubillas, A. M.; Unterkofler, S.; Euser, T. G.; Etzold, B. J. M.; Jones, A. C.; Sadler, P. J.; Wasserscheid, P.; Russell, P. St. J. Photonic crystal fibres for chemical sensing and photochemistry. Chem. Soc. Rev. 2013, 42, 8629-8648.

(10) Ritari, T.; Tuominen, J.; Ludvigsen, H.; Petersen, J.; Sørensen, T.; Hansen, T.; Simonsen, H. Gas sensing using air-guiding photonic bandgap fibers. Opt. Express 2004, 12, 4080-4087.

(11) Cordeiro, C. M. B.; dos Santos, E. M.; Brito Cruz, C. H.; de Matos, C. J.; Ferreira, D. S. Lateral access to the holes of photonic crystal fibers - selective filling and sensing applications. Opt. Express 2006, 14, 8403-8412. 
(12) Reader-Harris, P.; Di Falco, A. Functional Metamaterials for Lab-on-Fiber. In Labon-Fiber Technology; Cusano, A., Consales, M., Crescitelli, A., Ricciardi, A., Eds.; Springer Series in Surface Sciences; Springer: Geneva, 2015; Vol. 56, pp 111-132.

(13) Consales, M.; Pisco, M.; Cusano, A. Lab-on-fiber technology: a new avenue for optical nanosensors. Photonic Sensors 2012, 2, 289-314.

(14) Smythe, E. J.; Dickey, M. D.; Whitesides, G. M.; Capasso, F. A technique to transfer metallic nanoscale patterns to small and non-planar surfaces. ACS Nano 2009, 3, 5965.

(15) Ricciardi, A.; Consales, M.; Quero, G.; Crescitelli, A.; Esposito, E.; Cusano, A. Labon-Fiber devices as an all around platform for sensing. Opt. Fiber Technol. 2013, 19, $772-784$.

(16) Di Falco, A.; Ploschner, M.; Krauss, T. F. Flexible metamaterials at visible wavelengths. New J. Phys. 2010, 12, 113006.

(17) Lipomi, D. J.; Martinez, R. V.; Kats, M. A.; Kang, S. H.; Kim, P.; Aizenberg, J.; Capasso, F.; Whitesides, G. M. Patterning the tips of optical fibers with metallic nanostructures using nanoskiving. Nano Lett. 2011, 11, 632-636.

(18) Di Falco, A.; Zhao, Y.; Alù, A. Optical metasurfaces with robust angular response on flexible substrates. Appl. Phys. Lett. 2011, 99, 163110.

(19) Reader-Harris, P.; Ricciardi, A.; Krauss, T. F.; Di Falco, A. Optical guided mode resonance filter on a flexible substrate. Opt. Express 2013, 21, 1002-1007.

(20) Kolle, M.; Zheng, B.; Gibbons, N.; Baumberg, J. J.; Steiner, U. Stretch-tuneable dielectric mirrors and optical microcavities. Opt. Express 2010, 18, 4356-4364.

(21) Cho, H.; Kim, J.; Park, H.; Won Bang, J.; Seop Hyun, M.; Bae, Y.; Ha, L.; Yoon Kim, D.; Min Kang, S.; Jung Park, T. et al. Replication of flexible polymer membranes 
with geometry-controllable nano-apertures via a hierarchical mould-based dewetting. Nat. Commun. 2014, 5, 3137.

(22) Pryce, I. M.; Aydin, K.; Kelaita, Y. A.; Briggs, R. M.; Atwater, H. A. Highly strained compliant optical metamaterials with large frequency tunability. Nano Lett. 2010, 10, $4222-4227$.

(23) Chanda, D.; Shigeta, K.; Gupta, S.; Cain, T.; Carlson, A.; Mihi, A.; Baca, A. J.; Bogart, G. R.; Braun, P.; Rogers, J. A. Large-area flexible 3D optical negative index metamaterial formed by nanotransfer printing. Nat. Nanotechnol. 2011, 6, 402-407.

(24) Wang, S. S.; Magnusson, R. Theory and applications of guided-mode resonance filters. Appl. Opt. 1993, 32, 2606-2613.

(25) Fehrembach, A.-L.; Talneau, A.; Boyko, O.; Lemarchand, F.; Sentenac, A. Experimental demonstration of a narrowband, angular tolerant, polarization independent, doubly periodic resonant grating filter. Opt. Lett. 2007, 32, 2269-2271.

(26) Sakat, E.; Héron, S.; Bouchon, P.; Vincent, G. Metal-dielectric bi-atomic structure for angular-tolerant spectral filtering. Opt. Lett. 2013, 38, 425-427.

(27) Kildishev, A. V.; Boltasseva, A.; Shalaev, V. M. Planar photonics with metasurfaces. Science 2013, 339, 1232009.

(28) Gao, H.; Zhou, W.; Odom, T. W. Plasmonic Crystals: A Platform to Catalog Resonances from Ultraviolet to Near-Infrared Wavelengths in a Plasmonic Library. Adv. Funct. Mater. 2010, 20, 529-539.

(29) Enkrich, C.; Wegener, M.; Linden, S.; Burger, S.; Zschiedrich, L.; Schmidt, F.; Zhou, J. F.; Koschny, T.; Soukoulis, C. Magnetic Metamaterials at Telecommunication and Visible Frequencies. Phys. Rev. Lett. 2005, 95, 203901. 
(30) Shambat, G.; Provine, J.; Rivoire, K.; Sarmiento, T.; Harris, J.; Vučković, J. Optical fiber tips functionalized with semiconductor photonic crystal cavities. Appl. Phys. Lett. 2011, 99, 191102.

(31) Yan, H.; Gu, C.; Yang, C.; Liu, J.; Jin, G.; Zhang, J.; Hou, L.; Yao, Y. Hollow Core Photonic Crystal Fiber Surface Enhanced Raman Probe. Proc. SPIE 2007, 6433, 643307.

(32) Yang, X.; Shi, C.; Newhouse, R.; Zhang, J. Z.; Gu, C. Hollow-Core Photonic Crystal Fibers for Surface-Enhanced Raman Scattering Probes. Int. J. Opt. 2011, 2011, 1-11.

(33) Pfeiffer, C.; Grbic, A. Cascaded metasurfaces for complete phase and polarization control. Appl. Phys. Lett. 2013, 102, 231116.

(34) Pfeiffer, C.; Emani, N. K.; Shaltout, A. M.; Boltasseva, A.; Shalaev, V. M.; Grbic, A. Efficient light bending with isotropic metamaterial huygens' surfaces. Nano Lett. 2014, 14, 2491-2497.

(35) Kang, D.; Martinez, R. V.; Whitesides, G. M.; Tearney, G. J. Miniature grating for spectrally-encoded endoscopy. Lab Chip 2013, 13, 1810-1816. 


\section{Graphical TOC Entry}

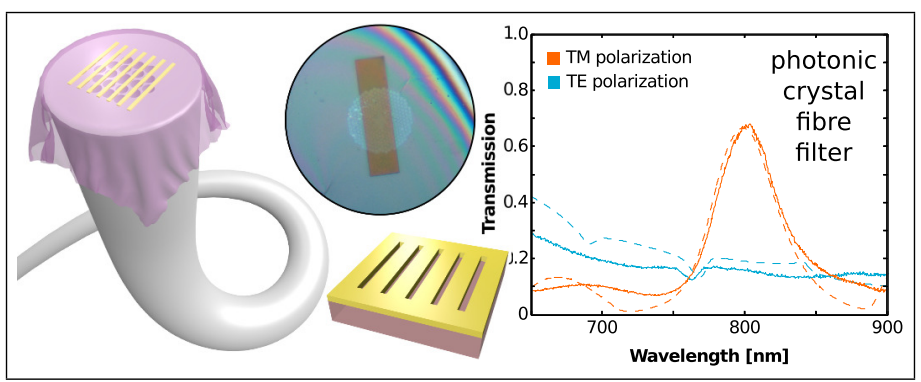

